\title{
Altered intrinsic regional brain spontaneous activity and subjective sleep quality in patients with chronic primary insomnia: a resting-state fMRI study
}

\author{
This article was published in the following Dove Press journal: \\ Neuropsychiatric Disease and Treatment \\ 14 November 2014 \\ Number of times this article has been viewed
}

\section{Xi-Jian Dai ${ }^{1,2}$ \\ De-Chang Peng' \\ Hong-Han Gong' \\ Ai-Lan Wan ${ }^{3}$ \\ Xiao $\mathrm{Nie}^{1}$ \\ Hai-Jun Li' \\ Yi-Xiang J Wang ${ }^{2}$}

'Department of Radiology, The First Affiliated Hospital of Nanchang University, Nangchang, Jiangxi, People's Republic of China; ${ }^{2}$ Department of Imaging and Interventional Radiology, Prince of Wales Hospital, The Chinese University of Hong Kong, Shatin, Hong Kong; ${ }^{3}$ Department of Psychosomatic Medicine, the First Affiliated Hospital of Nangchang University, Nangchang, Jiangxi, People's Republic of China
Correspondence: Hong-Han Gong Department of Radiology, The First Affiliated Hospital of Nanchang University, No 17, YongWai Zheng Street, DongHu District, Nanchang 330006, Jiangxi, People's Republic of China Tel +86 79l 88692582

Email honghan_gong@sina.com; daixjdoctor@126.com
Study objective: To prospectively explore the underlying regional homogeneity (ReHo) brain-activity deficit in patients with chronic primary insomnia (PCPIs) and its relationship with clinical features.

Design: The ReHo method and Statistical Parametric Mapping 8 software were used to evaluate whether resting-state localized brain activity was modulated between PCPIs and good sleepers (GSs), and correlation analysis between altered regional brain areas and clinical features was calculated.

Patients and participants: Twenty-four PCPIs (17 females, seven males) and 24 ( 12 females, 12 males) age-, sex-, and education-matched GSs.

Measurements and results: PCPIs disturbed subjective sleep quality, split positive mood, and exacerbated negative moods. Compared with GSs, PCPIs showed higher ReHo in left fusiform gyrus, and lower ReHo in bilateral cingulate gyrus and right cerebellum anterior lobe. Compared with female GSs, female PCPIs showed higher ReHo in the left fusiform gyrus and right posterior cingulate, and lower ReHo in the left cerebellum anterior lobe and left superior frontal gyrus. Compared with male GSs, male PCPIs showed higher ReHo in the right temporal lobe and lower ReHo in the bilateral frontal lobe. The fusiform gyrus showed strong positive correlations and the frontal lobe showed negative correlations with the clinical measurements.

Conclusion: The ReHo analysis is a useful noninvasive imaging tool for the detection of cerebral changes and the indexing of clinical features. The abnormal spontaneous activity areas provided important information on the neural mechanisms underlying emotion and sleep-quality impairment in PCPIs.

Keywords: insomnia, regional homogeneity, functional magnetic resonance imaging, sex difference, sleep disorder, blood oxygen level-dependent, mood disorder

\section{Introduction}

Sleep may allow the removal of free radicals accumulated in the brain during wakefulness ${ }^{1}$ and protect the structural stability of neuronal synapses. After sleep, the tired nerve cells and the biological characteristics of long-distance signal transmission recover normal physiological function. In general, a good sleep may be considered as a strong predictor of a good health. ${ }^{2,3}$ Conversely, a disturbed and/or interrupted sleep would adversely affect cognitive function, academic performance, and attentive ability, ${ }^{4-6}$ and is associated with poor emotional and physical health, ${ }^{7}$ conduct problems, and substance use, ${ }^{8,9}$ in both sexes and at all ages. ${ }^{7,10}$ Chronic primary insomnia (CPI), one of the most prevalent health complaints worldwide, is expected to further increase 
with the aging of society. ${ }^{11}$ Patients with CPI (PCPIs) who underwent chronically disturbed sleep and sleep loss, nonrefreshing sleep, and heightened arousal in bed ${ }^{12}$ frequently experience intrusive thoughts, which have been described as worrisome and negatively toned.

To date, limited work has been done to investigate CPI within a neuroscientific framework, and only a handful of studies have directly addressed the structural bases of CPI traits. A previous positron emission tomography study found that, compared with good sleepers (GSs), PCPIs displayed a relative increase in global cerebral metabolic rate for glucose utilization, both during sleep and when awake. ${ }^{13}$ A singlephoton emission computed tomography study found that PCPIs displayed a significant decrease in regional cerebral blood flow in frontal, parietal, and occipital cortices and basal ganglia during sleep. ${ }^{14}$ A number of voxel-based morphometry studies have demonstrated several significantly changed gray matter concentrations (GMCs) in the frontal lobe, temporal lobe, hippocampus, and cerebellum. ${ }^{15,16}$ Riemann et $\mathrm{al}^{17}$ found reduced hippocampal volumes, but none of other regions of interest analyzed revealed volume differences between PCPIs and GSs. Conversely, other studies found no significant between-group differences (eg, hippocampus) in any of the investigated brain morphometry variables. ${ }^{18,19}$ Although these studies focus on the brain structural changes in PCPIs, few resting-state functional magnetic resonance imaging (rs-fMRI) studies have been conducted to examine neural bases of PCPI brain traits, few studies take mood deficits (eg, depression, anxiety) into consideration, and still too many areas of dispute have been left unresolved. Therefore, it is important to explore the potential health effects of CPI on patients, especially the microscopic functional and emotional change aspects.

Previous studies have demonstrated that the synchronous activity of neurons is conducive to the integration and coordination of information processing in the brain, ${ }^{20}$ but altering neuronal synchrony may lead to the deterioration of information processing speed and efficiency, resulting in dysfunction. ${ }^{21}$ The regional homogeneity (ReHo) method, a widely used rs-fMRI measure, assesses the functional consistency and similarity for each individual by calculating Kendall's coefficient concordance (KCC) of the time series of a given voxel with its nearest neighbors. The ReHo method is based on a data-driven approach and thus does not require onset time of stimulus and prior knowledge, and it has good testretest reliability. It can be used to evaluate resting-state brain activities based on the hypothesis that the hemodynamic characteristics of each voxel are similar within a functional cluster and that there is dynamic synchronization of voxels within a given cluster. ${ }^{22-24}$ Therefore, the simple calculation and reliable characterization of the ReHo method makes it a potentially useful tool for rs-fMRI data analysis, as a measure for investigating intrinsic brain activities during the resting-state condition and whether the local synchronization of spontaneous brain activities is associated with PCPI traits.

Recently, ReHo has been successfully used to explore functional modulations and to characterize pathophysiological changes in patients with sleep problems, such as obstructive sleep apnea ${ }^{25}$ and sleep deprivation, ${ }^{24}$ and various neuropsychiatric disorders related to mood/ emotional changes, such as unhappy individuals, ${ }^{26}$ social anxiety disorders, ${ }^{27}$ panic disorder, ${ }^{28}$ early life stress ${ }^{29}$ and depression. ${ }^{30}$ However, it has not yet been used to explore pathophysiological changes in PCPIs.

On the basis of the studies reviewed above, we hypothesized that, compared with GSs, PCPIs would exhibit abnormal ReHo activities in at least parts of certain brain areas, including the prefrontal lobe, temporal lobe, hippocampus, and cerebellum, together with emotional changes. To test the hypothesis, the present prospective study is the first to characterize and compare ReHo differences between PCPIs and GSs using rs-fMRI to understand the underlying effect of CPI patients on brain function.

\section{Materials and methods \\ Subjects}

Twenty-four PCPIs (17 females and seven males) who had sleep onset and/or maintenance insomnia were recruited from the Psychiatry Department of The First Affiliated Hospital of Nanchang University. All subjects were evaluated with an unstructured clinical interview for history of medical and sleep disorders and with a structured interview (the Structured Clinical Interview for Diagnostic and Statistical Manual of Mental Disorders, version 4 (DSM-IV) administered by an experienced psychiatrist) to document life history of psychiatric disorders.

All PCPIs met the following criteria: 1) conformity to the definition of CPI by the International Classification of Sleep Disorders-2 (ICSD-2); 2) insomnia lasting $\geq 5$ months; 3 ) a Pittsburgh Sleep Quality Index (PSQI) score $\geq 7$; 4) had a sleep diary for at least 2 weeks' duration; 5) right-handedness; 6) no history of inborn or other acquired diseases such as psychiatric disorder, hypertension, diabetes mellitus, coronary artery disease, no addictions such as heroin addiction, smoking addiction, or alcohol addiction, and no other sleep disorders, including hypersomnia, parasomnia, sleep-related 
breathing disorder, sleep-related movement disorder, or circadian rhythm sleep disorder; 7) no foreign implants in the body; and 8) moderate body shape and weight.

Twenty-four GSs (12 females and 12 males) who were age-, sex-, and education status-matched to PCPIs were recruited for this study. All GSs met the following criteria: 1) good sleeping habits and good sleep onset and/or maintenance; 2) regular dietary habits; 3 ) no history of swing shift, shift work, or sleep complaints; 4) no consumption of stimulants, medications, tea, or coffee for at least 3 months prior to the study; 5) PSQI score $<5$, Hamilton Depression Rating Scale (HAMD) and Hamilton Anxiety Rating Scale (HAMA) scores $<7$. All GSs also met criteria 5-8 above for PCPIs.

\section{Research design and procedures}

All volunteers were asked to wear a Fitbit Flex tracker (http:// help.fitbit.com); PCPIs wore the tracker for 2 consecutive nights to evaluate their sleep status, and GSs wore it for 1 week to exclude poor sleepers. Meanwhile, the total sleep time, sleep onset latency, and sleep efficiency were recorded. Before the tests, PCPIs who had consumed psychoactive medications (eg, benzodiazepine) were asked to stop taking any medications for 48-96 hours prior to and during the study. Another eight patients were first-time visitors and had never taken any psychoactive medications before. All volunteers were asked to empty their feces and urine, and to rest quietly for 30 minutes before the rs-fMRI scans. The volunteers were instructed to wear black blinders and sponge earplugs, and fix the head, to avoid audiovisual stimulus during the rs-fMRI scans. They were told to relax and not to think of anything, and not to fall asleep in particular. A simple questionnaire was administered immediately after the scan to ask whether the subjects were awake during the scan. The data of the subjects who were asleep during the scan were excluded. This study was approved by The Human Research Ethics Committee of the First Affiliated Hospital of Nanchang University. All volunteers participated voluntarily and were informed of the purposes, methods, and the potential risks, and all signed an informed consent form.

\section{Questionnaires}

All volunteers were asked to complete a number of questionnaires, including the PSQI, ${ }^{31}$ Insomnia Severity Index (ISI), ${ }^{32}$ HAMD $^{33}$ HAMA, $^{34}$ Self-Rating Scale of Sleep (SRSS) and Profile of Mood States (POMS). ${ }^{35,36}$ The POMS questionnaire contains seven indexes in which five (nervousness, wrath, fatigue, depression, and confusion) reflect negative emotion and two (energy and self-esteem) reflect positive emotion.

\section{fMRI parameters}

fMRI scanning was performed on a 3-Tesla magnetic resonance scanner (Siemens, Germany). High-resolution T1-weighted images were acquired with a three-dimensional spoiled gradient-recalled sequence in an sagittal orientation: 176 images (repetition time $=1,900 \mathrm{~ms}$, echo time $=2.26 \mathrm{~ms}$, thickness $=1.0 \mathrm{~mm}$, gap $=0.5 \mathrm{~mm}$, acquisition matrix $=256 \times 256$, field of view $=250 \mathrm{~mm}$ $\times 250 \mathrm{~mm}$, flip angle $=9^{\circ}$ ) were obtained. Finally, 240 functional images (repetition time $=2,000 \mathrm{~ms}$, echo time $=30 \mathrm{~ms}$, thickness $=4.0 \mathrm{~mm}$, gap $=1.2 \mathrm{~mm}$, acquisition matrix $=64 \times 64$, flip angle $=90^{\circ}$, field of view $=220 \mathrm{~mm}$ $\times 220 \mathrm{~mm}, 29$ axial slices with Gradient-Recalled EchoPlanar Imaging pulse sequence) covering the whole brain were obtained.

\section{fMRI data analysis}

Functional data were checked by MRIcro software (www. MRIcro.com) to exclude defective data. The first ten time points of the functional images were discarded due to the possible instability of the initial fMRI signal and the participants' adaptation to the scanning environment. On the basis of MATLAB2010a (Mathworks, Natick, MA, USA), the rest of the data preprocessing was performed by DPARSFA (http://rfmri.org/DPARSF) software, including Digital Imaging and Communications in Medicine (DICOM) standards for form transformation, slice timing, head motion correction, and spatial normalization. Motion time courses were obtained by estimating the values for translation (millmeters) and rotation (degrees) for each subject. The participants who had more than $1.5 \mathrm{~mm}$ maximum displacement in $\mathrm{x}, \mathrm{y}$, or $\mathrm{z}$ and $1.5^{\circ}$ of angular motion during the whole fMRI scans would be rejected. After head-motion correction, the fMRI images were spatially normalized to the Montreal Neurological Institute (MNI) space using the standard Echo-Planar Imaging template in Statistical Parametric Mapping 8 (SPM8, http://www.fil.ion.ucl.ac.uk/ spm) software and resampling the images at a resolution of $3 \mathrm{~mm} \times 3 \mathrm{~mm} \times 3 \mathrm{~mm}$. After preprocessing, the time series for each voxel were temporally bandpass filtered (0.01-0.08 $\mathrm{Hz}$ ) and linearly detrended to reduce low-frequency drift and physiological high-frequency respiratory and cardiac noise and time series linear detrending. The Friston six head motion parameters obtained by rigid body correction were used to regress out head motion effects based on recent work showing that higher-order models were more effective in removing head-motion effects. ${ }^{37,38}$ Linear regression was also applied to remove other sources of spurious covariates 
along with their temporal derivatives, including the signal from a ventricular region of interest, and the signal from a region centered in the white matter. ${ }^{39}$ Of note, the global signal was not regressed out in the present data, as in Guo et $\mathrm{al}^{40}$ for the reason that it is still controversial to remove the global signal in the preprocessing of resting-state data. ${ }^{39,41}$

Individual ReHo maps were generated for each dataset by calculating the $\mathrm{KCC}$ of the time series of a given voxel with those of its nearest neighbors (26 voxels) in a voxel-wise analysis, as in our previous study. ${ }^{24}$ To reduce the influence of individual variations in the KCC value, normalization of ReHo maps was done by dividing the $\mathrm{KCC}$ among each voxel by the averaged $\mathrm{KCC}$ of the whole brain. The resulting fMRI data were then spatially smoothed with a Gaussian kernel of $6 \times 6 \times 6 \mathrm{~mm}^{3}$ full-width at halfmaximum (FWHM).

\section{Statistical analysis}

For behavior performance, two-sample Student's $t$-test was used for continuous data, and chi-square test was used for categorical data. All the results are quoted as two-tailed $P$-values. $P<0.05$ was considered statistically significant. All the statistical analyses were performed using IBM SPSS version 21.0 statistical software.

For fMRI data, two-sample Student's $t$-tests were used to assess the differences between two groups, with age, years of education, and/or sex as nuisance covariates of no interest. A corrected significance level of individual voxel $P<0.01$ and $V \geq 1,080 \mathrm{~mm}^{3}$, using an AlphaSim corrected cluster threshold of $P<0.05$, was used to determine the statistical significance.

\section{Brain-behavior correlation analysis}

Based on ReHo findings, the brain regions that demonstrated significant differences between groups were identified. These regions were classified as regions of interest and saved as masks with REST software. For each region of interest, the mean ReHo value was extracted by averaging ReHo values over all voxels for each PCPI. Finally, the mean ReHo values were entered into IBM SPSS 21.0 software to calculate their correlations with the behavioral performances.

\section{Results}

\section{Behavioral results}

Compared with GSs, PCPIs demonstrated worse subjective sleep estimates, respectively, as measured by PSQI(PSQI score: 15.6 \pm 2.1 versus $2.3 \pm 0.8, P=0.001$; time in bed: $8.4 \pm 1.2$ hours versus $8.6 \pm 0.5$ hours, $P=0.591$; total sleep time: $3.7 \pm$ 1.1 hours versus $7.6 \pm 0.6$ hours, $P<0.001$; sleep efficiency:
$44.7 \% \pm 15.7 \%$ versus $89.1 \% \pm 59.0 \%, P<0.001)$ and SelfRating Scale of Sleep (SRSS) (35.3 \pm 4.7 versus 15.3 \pm 2.0 , $P<0.001)$, and disturbed mood state as measured by HAMD (10.5 \pm 5.3 versus $1.9 \pm 1.2, P<0.001)$, HAMA $(8.8 \pm 3.1$ versus 1.5 $\pm 1.2, P<0.001$ ), and POMS (POMS total score: $115.2 \pm 26.3$ versus $84.7 \pm 4.5, P<0.001$; nervousness: $8.0 \pm 5.5$ versus $1.9 \pm 0.9, P<0.001$; wrath: $6.1 \pm 5.1$ versus $1.9 \pm 0.9$, $P<0.001$; fatigue: $6.2 \pm 4$.7 versus $1.9 \pm 0.9, P<0.001$; depression: $5.0 \pm 4.6$ versus $0.6 \pm 0.7, P<0.001$; confusion: $6.6 \pm 3.3$ versus $3.1 \pm 1.5, P<0.001$; energy: $9.4 \pm 4.9$ versus $15.0 \pm 1.2$, $P<0.001$; self-esteem: $7.3 \pm 3.6$ versus 9.8 $\pm 2.4, P=0.012$ ). The details are presented in Tables 1 and 2.

Female PCPIs (PCPI-Fs) compared with female GSs (GS-Fs), as well as male PCPIs (PCPI-Ms) compared with male GSs (GS-Ms), showed significantly disturbed subjective sleep estimates as measured by PSQI $(P<0.05)$ and SRSS $(P<0.05)$, and disturbed mood state as measured by HAMD $(P<0.05)$, HAMA $(P<0.05)$, and POMS $(P<0.05)$. Compared with PCPI-Ms, PCPI-Fs only showed significantly longer total sleep time $(3.3 \pm 1.1$ hours versus $4.4 \pm 0.9$ hours, $P=0.032)$ and higher sleep efficiency $(39.0 \% \pm 13.1 \%$ versus $58.6 \% \pm 13.1 \%, P=0.003)$. The ISI score was $19.3 \pm 2.7$ for PCPIs, 19.1 \pm 2.9 for PCPI-Fs, and 19.9 \pm 2.1 for PCPI-Ms. The details are presented in Tables 1 and 2 .

\section{Behavioral correlations}

Duration of insomnia showed positive correlations with total PSQI score $(r=0.539, P=0.007)$, POMS nervousness ( $r=0.440, P=0.032)$, wrath $(r=0.414, P=0.045)$, depression ( $r=0.458, P=0.024)$, confusion $(r=0.575, P=0.003)$, total score of five negative indexes $(r=0.512, P=0.011)$, and POMS total score $(r=0.454, P=0.026)$. PSQI displayed positive correlations with HAMD ( $r=0.620, P=0.001)$, HAMA ( $r=0.412, P=0.045)$, POMS confusion $(r=0.0 .410, P=0.047)$, and POMS total score $(r=0.407, P=0.048)$, and negative correlations with POMS energy ( $r=-0.466, P=0.022)$, self-esteem $(r=-0.427, P=0.037)$ and total score of two positive indexes $(r=-0.470, P=0.021)$. HAMD and HAMA each showed positive correlation with POMS nervousness, wrath, depression, confusion, total score of five negative indexes, and POMS total score, and negative correlation with POMS energy, self-esteem, and total score of two positive indexes. ISI score showed positive correlation with SRSS score $(r=0.511, P=0.011)$. Total POMS score of five negative indexes showed positive correlation with each of the five negative indexes, and negative correlation with energy, self-esteem, total score of two positive indexes and POMS score. Total POMS score of two positive indexes showed 


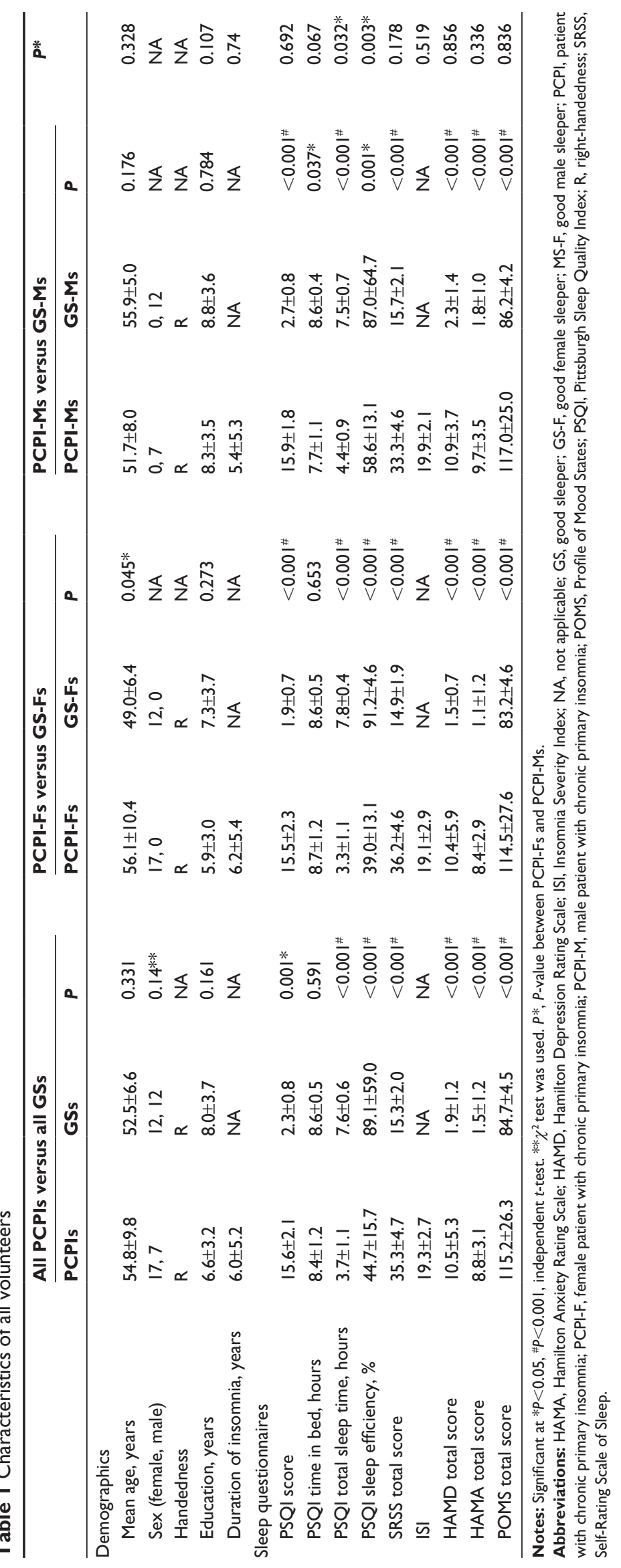




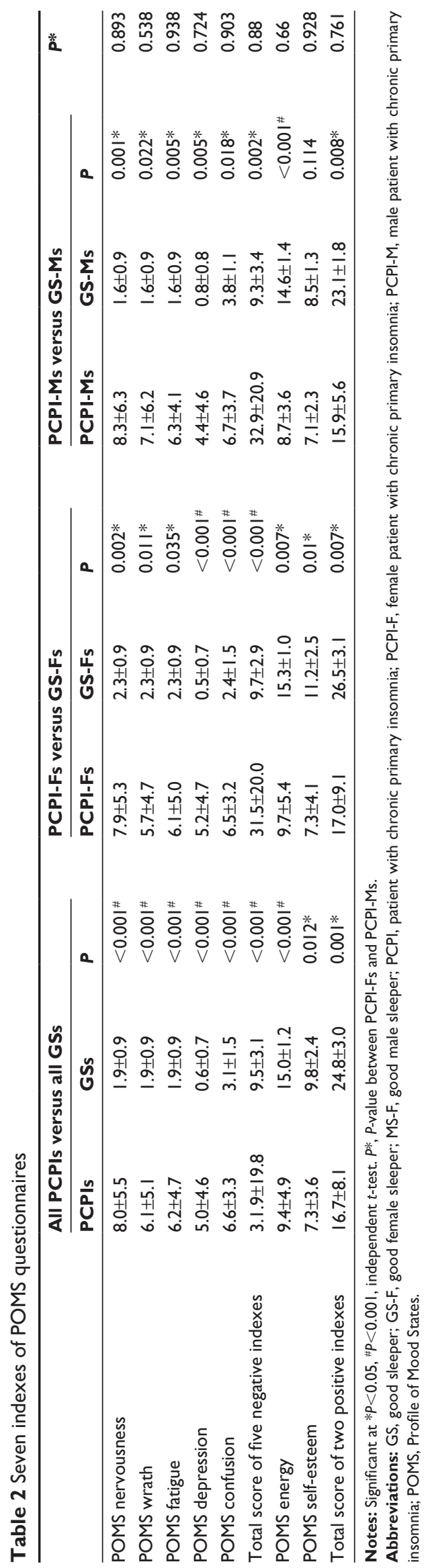

positive correlations with energy and self-esteem, and negative correlations with the five POMS negative indexes and their total score.

\section{ReHo and brain-behavior correlation All PCPIs compared with all GSs}

Compared with GSs, PCPIs showed significantly higher ReHo in the left fusiform gyrus of the temporal lobe (Brodmann area [BA]37), and lower ReHo in the bilateral cingulate gyrus (BA23, BA24) and right cerebellum anterior lobe (lingual, culmen). In PCPIs, the observed bilateral cingulate gyrus showed significant negative correlation with HAMA ( $r=-0.436, P=0.033)$, but the right cerebellum anterior lobe did not show any correlations with behavioral performance. The left fusiform gyrus showed significant positive correlations with duration of insomnia ( $r=0.443, P=0.03)$, PSQI score $(r=0.410, P=0.047)$, SRSS score $(r=0.477, P=0.018)$, POMS nervousness $(r=0.405$, $P=0.05)$, fatigue $(r=0.486, P=0.016)$, depression $(r=0.711$, $P<0.001)$, confusion $(r=0.666, P<0.001)$, total score of five negative indexes $(r=0.603, P=0.002)$ and POMS total score ( $r=0.576, P=0.003$ ), and negative correlation with POMS energy $(r=-0.416, P=0.043)$. The details are presented in Table 3 and Figure 1.

\section{PCPI-Fs compared with GS-Fs}

Compared with GS-Fs, PCPI-Fs showed higher ReHo in the left fusiform gyrus of the temporal lobe (BA37) and right cluster of the posterior cingulate gyrus, occipital lobe, and parahippocampal gyrus (BA17, BA18, BA27, BA30), and lower ReHo in the left cerebellum anterior lobe (culmen, declive, nodule) and left superior frontal gyrus (BA10, BA11). The details are presented in Table 3 and Figure 2A.

In PCPI-Fs, only the higher ReHo in the left fusiform gyrus showed significant positive correlation with HAMA score $(r=0.504, P=0.039)$. The details are presented in Figure 2B.

\section{PCPI-Ms compared with GS-Ms}

Compared with GS-Ms, PCPI-Ms showed significantly higher ReHo in the right temporal lobe (middle temporal gyrus, inferior temporal gyrus; BA20, BA21), and lower $\mathrm{ReHo}$ in the left frontal lobe (superior frontal gyrus, middle frontal gyrus; BA6) and bilateral medial frontal gyrus (BA6). The details are presented in Table 3 and Figure 3A.

In PCPI-Ms, the left frontal lobe showed significant negative correlations with duration of insomnia $(r=-0.771$, $P=0.042)$ and PSQI score $(r=-0.848, P=0.016)$; the bilateral 


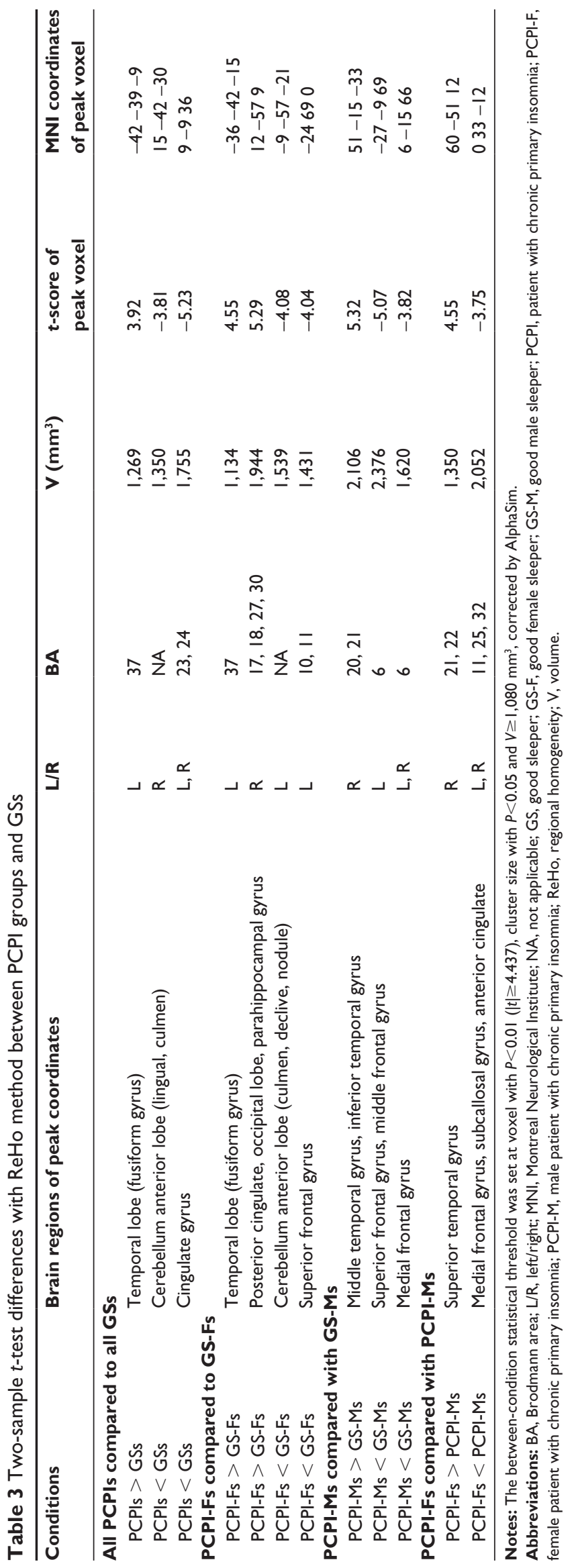

middle frontal gyrus showed significant negative correlation with PSQI score $(r=-0.762, P=0.046)$. However, the temporal lobe did not display significant correlations with any behavioral performance. The details are presented in Figure 3B.

\section{PCPI-Fs compared with PCPI-Ms}

Compared with PCPI-Ms, PCPI-Fs showed significantly higher ReHo in right superior temporal gyrus (BA21, BA22), and lower ReHo in the cluster of bilateral medial frontal gyrus, subcallosal gyrus and anterior cingulate (BA11, BA25, BA32). The details are presented in Table 3 and Figure 3C. The mean blood oxygenation level dependent (BOLD) signal value of the altered ReHo areas, as well as the different areas between other groups, as presented above, was extracted. The details of the BOLD signal value are presented in Figure 4A-D. But the altered ReHo areas didn't display any significant correlations with PSQI total sleep time and sleep efficiency in PCPI-Fs and PCPI-Ms.

\section{Discussion}

To our knowledge, this study is the first to evaluate whether resting-state local functional homogeneity was modulated by CPI utilizing an ReHo analysis and rs-fMRI approach. In our study, PSQI showed significant positive correlations with negative indexes and duration of insomnia and negative correlation with positive moods, suggesting that PSQI could be useful for indexing the duration of insomnia and mood state. Significant group differences in ReHo were observed within distributed brain regions over the temporal lobe, frontal lobe, cerebellum, and cingulate gyrus, along with disturbed subjective sleep estimates as measured by PSQI and SRSS, and disturbed mood state estimates as measured by HAMD, HAMA, and POMS, suggesting multiple clinical symptoms and brain dysfunctions were involved in PCPIs. In addition, we focused on the correlations between the ReHo differences and a number of questionnaires in CPI groups.

\section{Temporal cortex deficits}

PCPIs are hypervigilant and ruminative according to neurocognitive models, and have shown an excessive hyperarousal of the central nervous system (CNS) throughout the night in electrophysiological studies. ${ }^{42-44}$ In support of these findings, we found that the higher ReHo in temporal lobes showed excessive hyperarousal and development of synchronization with negative moods, duration of insomnia, and subjective sleep quality deficits, and negative correlation with positive moods in all PCPIs, PCPI-Ms, and PCPI-Fs compared with GSs. The fusiform gyrus is responsible for 

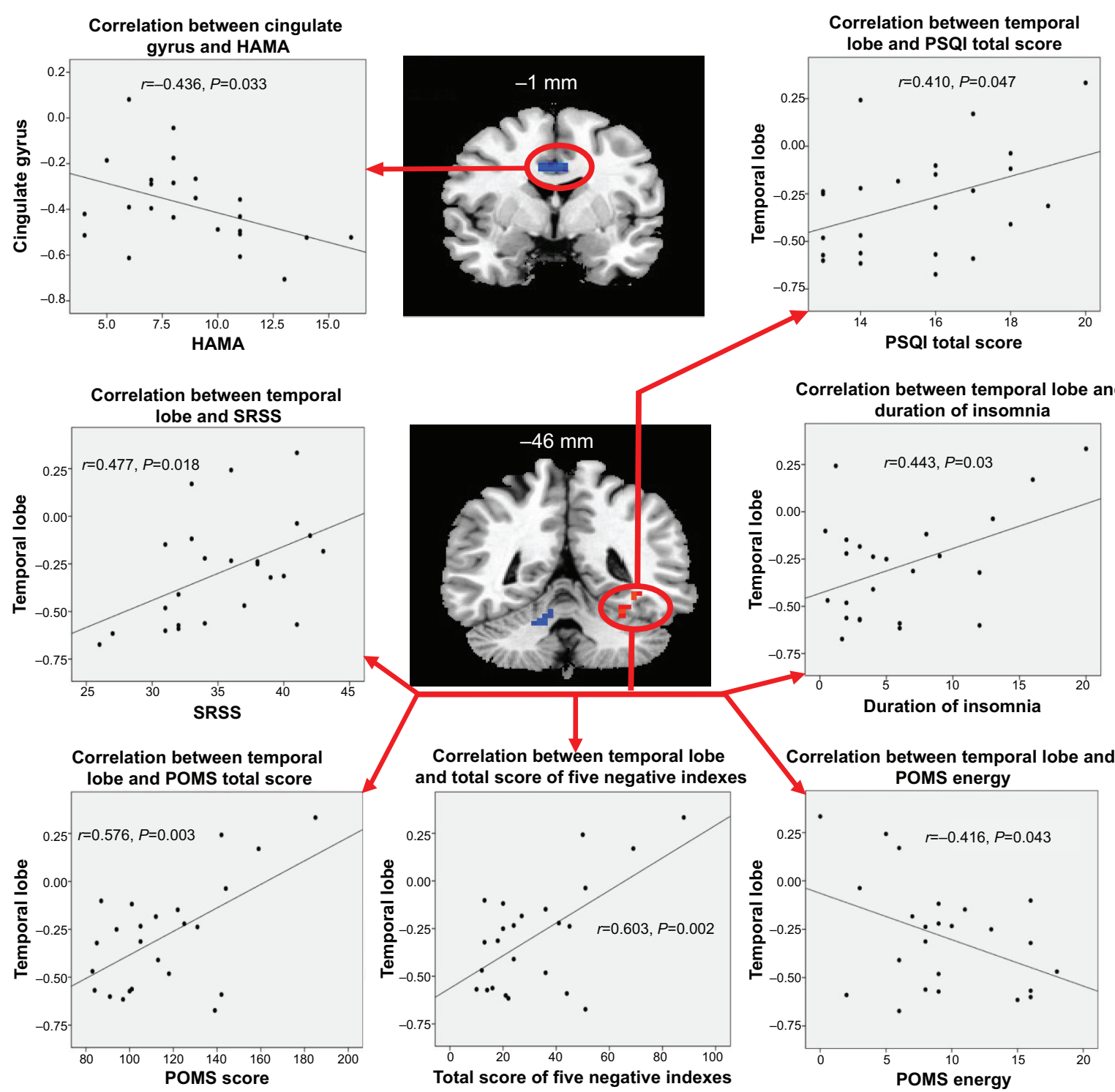

Correlation between temporal lobe and duration of insomnia
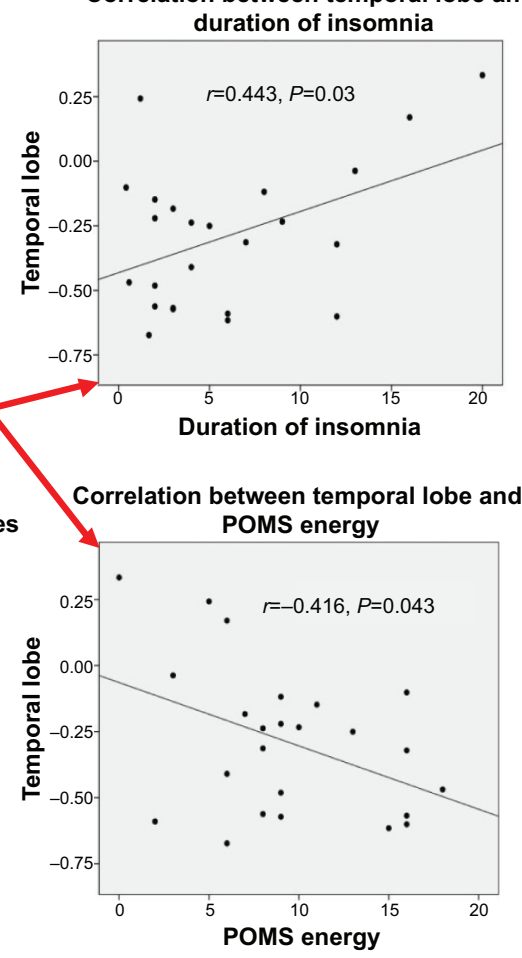

Figure I Various correlations for patients with chronic primary insomnia.

Notes: Compared with GSs, PCPIs show higher ReHo in the left fusiform gyrus of the temporal lobe, and lower ReHo in the cingulate gyrus and right cerebellum anterior lobe. The cingulate gyrus shows significant correlation with the HAMA. The fusiform gyrus shows significant correlations with PSQI score, SRSS score, duration of insomnia, POMS total score, total score of five negative indexes, and POMS energy. But the right cerebellum anterior lobe didn't find any correlations with the behavioral performance.

Abbreviations: HAMA, Hamilton Anxiety Rating Scale; GS, good sleep; PCPI, patient with chronic primary insomnia; POMS, Profile of Mood States; PSQI, Pittsburgh Sleep Quality Index; ReHo, regional homogeneity; SRSS, Self-Rating Scale of Sleep.

multimodal combined and face identification, because of which the fusiform gyrus may play a regulative role and be involved in mood. Our findings suggest that the temporal lobe (mainly in the fusiform gyrus) could be useful for indexing the extent of insomnia traits and mood state, and the hyperarousal reactivity of CPI may contribute to changes in increased activity in the temporal cortex.

\section{Prefrontal cortex deficits}

Altena et $\mathrm{al}^{45}$ found that PCPIs had a smaller volume of gray matter (GMV) in the left orbitofrontal cortex, strongly correlating with the subjective severity of insomnia.
Joo et $\mathrm{al}^{15}$ found that PCPIs showed significant reduction of GMC in left or right dorsolateral prefrontal cortices (right superior frontal gyrus, left middle frontal gyrus, and bilateral inferior frontal gyrus), and decreased GMV in medial frontal gyrus compared with GSs. Noh et al ${ }^{46}$ found that PCPIs had significantly lower scores on tests of attention and frontal-lobe function relative to GSs. In support of these findings, in the present study we found that the lower ReHo in the frontal gyrus was found both in PCPI-Ms compared with GS-Ms and in PCPI-Fs compared with GS-Fs. Furthermore, the PCPI-Fs showed more significant damage than PCPI-Ms. 

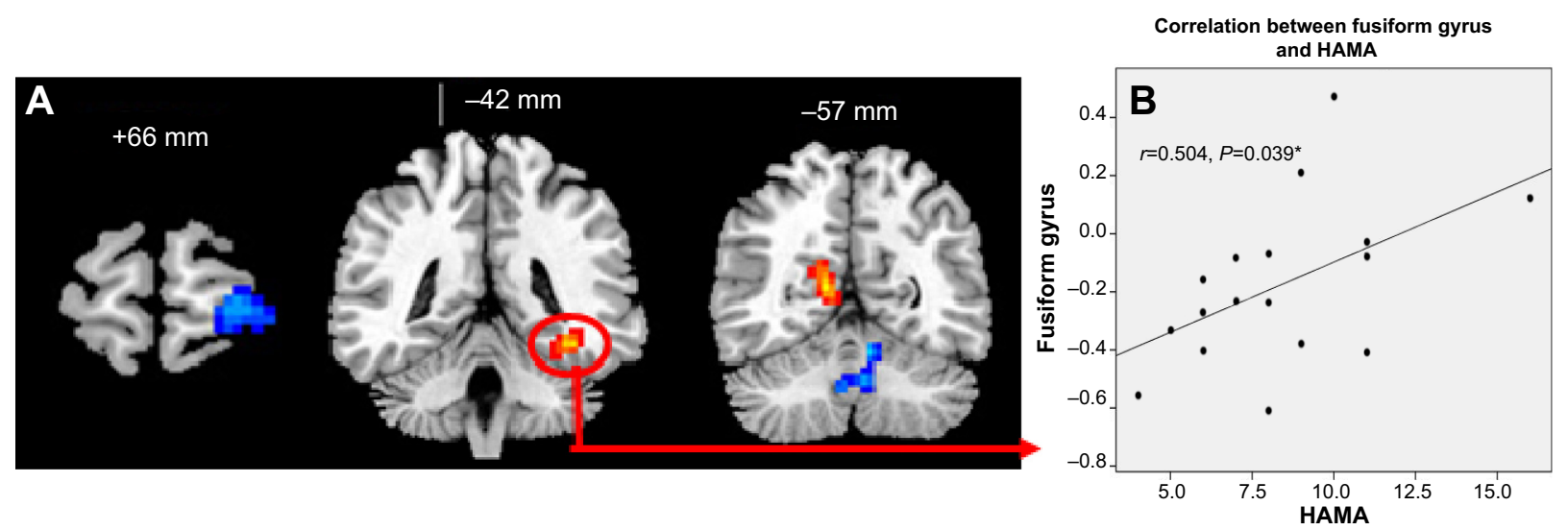

Figure 2 Altered ReHo areas between PCPI-Fs and GSs and its correlations with behavioral performances.

Notes: (A) Compared with GS-Fs, PCPI-Fs showed higher ReHo in the left fusiform gyrus and right posterior cingulate, and lower ReHo in the left cerebellum anterior lobe and left superior frontal gyrus. (B) Only the fusiform gyrus showed significant correlation with the HAMA. $* P<0.05$.

Abbreviations: HAMA, Hamilton Anxiety Rating Scale; GS-F, female good sleeper; PCPI-F, female patient with chronic primary insomnia; ReHo, regional homogeneity.

Converging evidence from lesion, neuroimaging, and electrophysiological data supports the view that the frontal cortex is a key component of the circuitry that implements both positive and negative affect. ${ }^{47}$ The middle frontal gyrus has been recognized as one key region associated with mood regulation ${ }^{48}$ and may be an important factor for the development of depressive symptoms in Parkinson's disease. ${ }^{49,50}$ In PCPI-Ms, the lower ReHo area in left superior frontal gyrus showed negative correlations with duration of insomnia and PSQI score, and the bilateral middle frontal gyrus showed
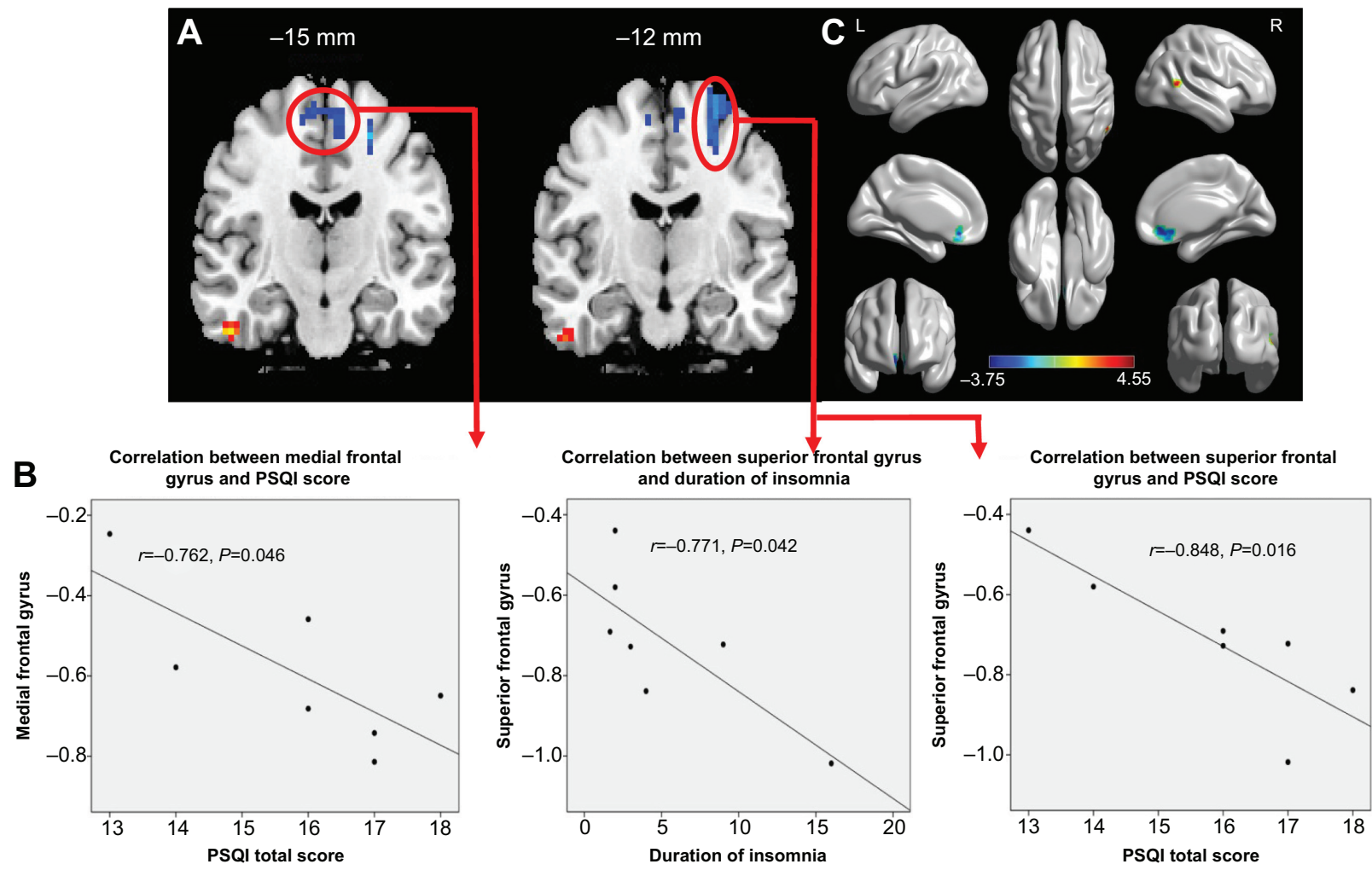

Figure 3 Patients with chronic primary insomnia compared with normal controls.

Notes: (A) Compared with GS-Ms, PCPI-Ms show higher ReHo in the right temporal lobe, and lower ReHo in the left superior frontal gyrus and bilateral medial frontal gyrus. (B) The medial frontal gyrus shows significant correlations with PSQI score, and the superior frontal gyrus shows significant correlations with duration of insomnia and PSQI score. (C) Compared with PCPI-Ms, PCPI-Fs show altered ReHo areas, including the right superior temporal gyrus and bilateral medial frontal gyrus.

Abbreviations: GS-M, male good sleeper; PCPI-F, female patient with chronic primary insomnia; PCPI-M, male patient with chronic primary insomnia; PSQI, Pittsburgh Sleep Quality Index; ReHo, regional homogeneity; L, left; R, right. 
A

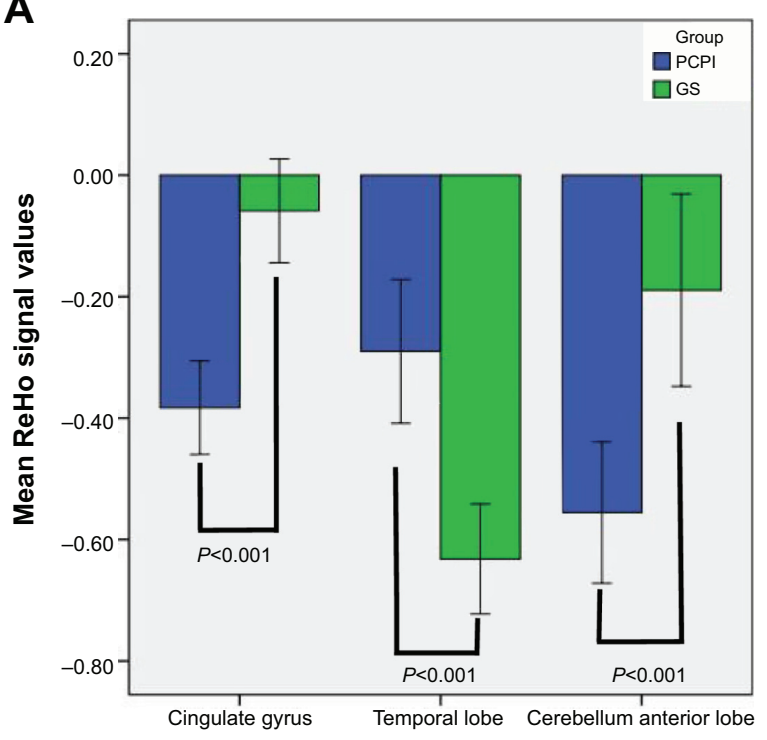

C

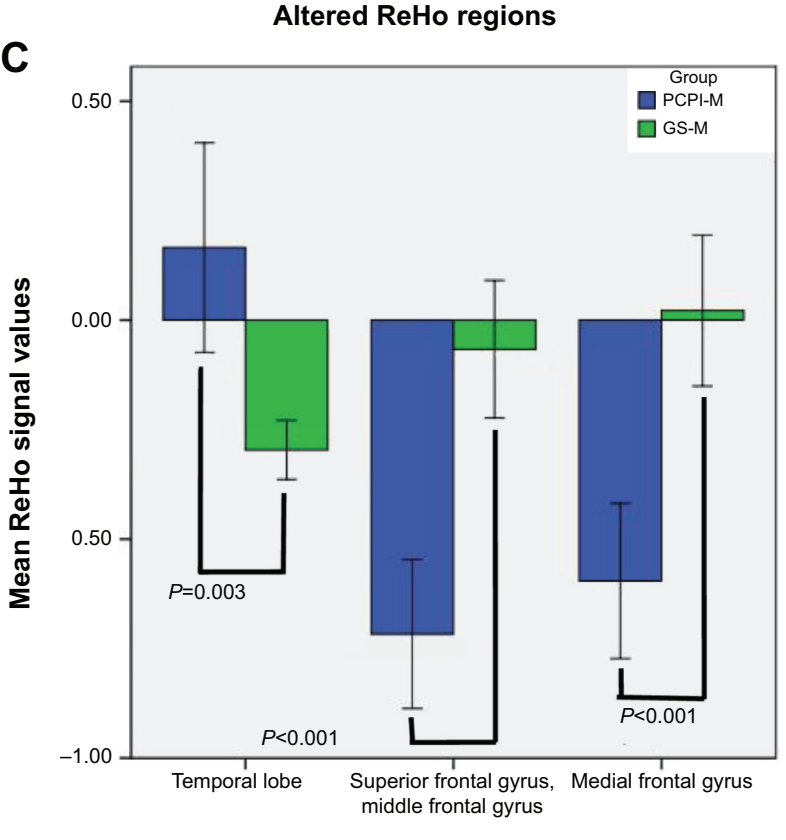

Altered ReHo regions
B

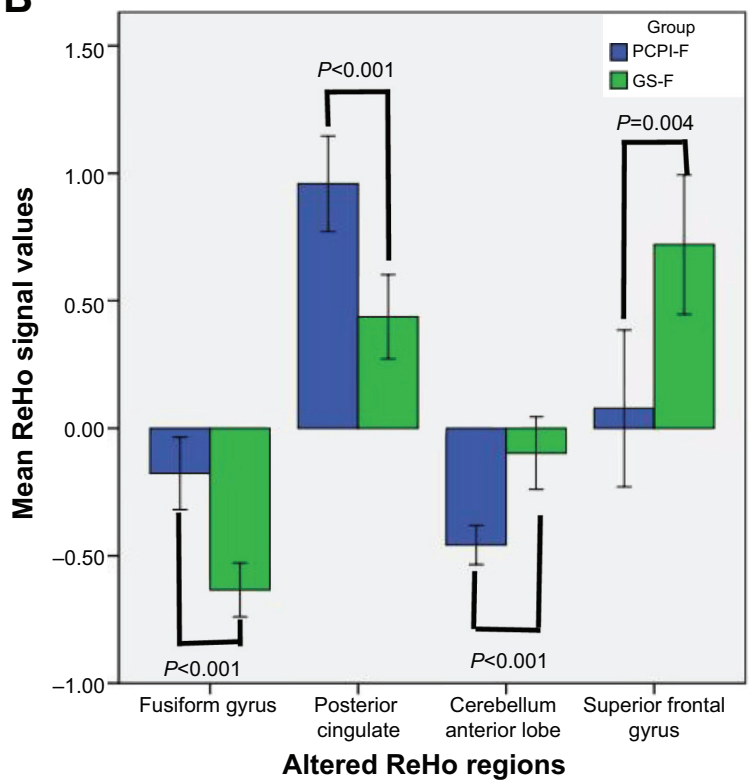

D

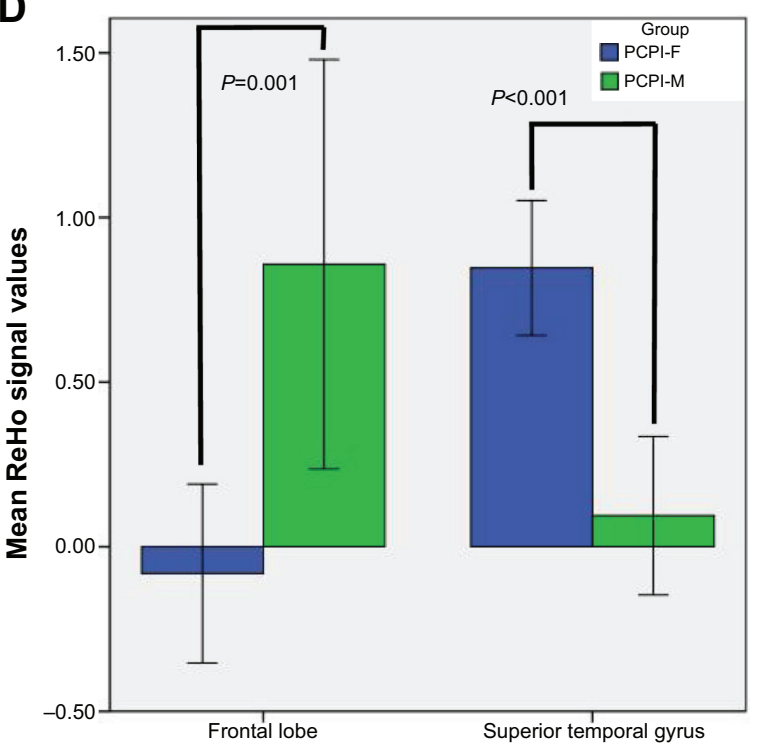

Altered ReHo regions

Figure 4 Mean ReHo signal values of altered regional brain areas.

Notes: (A) Compared with GSs, PCPIs showed altered ReHo in the cingulate gyrus $(-0.38 \pm 0.18$ versus $-0.06 \pm 0.20)$, temporal lobe $(-0.29 \pm 0.28$ versus $-0.63 \pm 0.21)$, and cerebellum anterior lobe $(-0.56 \pm 0.28$ versus $-0.19 \pm 0.38)$. (B) Compared with GS-Fs, PCPI-Fs showed altered ReHo in the fusiform gyrus $(-0.18 \pm 0.28$ versus $-0.63 \pm 0.17)$, posterior cingulate $(0.96 \pm 0.36$ versus $0.44 \pm 0.26)$, cerebellum anterior lobe $(-0.46 \pm 0.15$ versus $-0.10 \pm 0.22)$, and superior frontal gyrus $(0.08 \pm 0.60$ versus $0.72 \pm 0.43)$. (C) Compared with GS-Ms, PCPI-Ms showed altered ReHo in the temporal lobe $(0.17 \pm 0.26$ versus $-0.30 \pm 0.1 \mathrm{I})$, superior frontal gyrus/middle frontal gyrus $(-0.72 \pm 0.18$ versus $-0.07 \pm 0.25)$, and medial frontal gyrus $(-0.60 \pm 0.19$ versus $0.02 \pm 0.27)$. (D) Compared with PCPI-Ms, PCPI-Fs showed altered ReHo in the frontal lobe $(-0.08 \pm 0.53$ versus $0.86 \pm 0.67)$ and superior temporal gyrus $(0.85 \pm 0.40$ versus $0.09 \pm 0.26)$.

Abbreviations: GS, good sleeper; GS-F, good female sleeper; GS-M, good male sleeper; PCPI, patient with chronic primary insomnia; PCPI-F, female patient with chronic primary insomnia; PCPI-M, male patient with chronic primary insomnia; PSQI, Pittsburgh Sleep Quality Index; ReHo, regional homogeneity.

negative correlation with PSQI score. In addition, duration of insomnia and PSQI score showed positive correlations with negative mood indexes and negative correlations with positive mood indexes. That is to say, the dysfunction of the frontal gyrus in PCPI-Ms, together with mood disorder, had a trend to accentuate gradually with the growth of the decline in subjective sleep quality and the duration of insomnia. Thus, we presume that the effects of CPI, together with the structural damage reviewed above, result in abnormally synchronized neural activities in the frontal gyrus. This abnormal activity causes abnormal ReHo values and, consequently, results in cognitive and emotional dysfunction. In conclusion, 
ReHo analysis may be an early biomarker for detecting the altered brain activity in PCPIs and could be useful for indexing the clinical features.

\section{Cingulate cortex deficits}

Terroni et $\mathrm{al}^{51}$ have shown that cingulate cortex dysfunction may be a key reason for poststroke depression and workingmemory damage in stroke patients. The cingulate cortex, with greater metabolism relative to the whole brain activity, plays an important role in emotion processing ${ }^{52}$ and self-control, ${ }^{53}$ and is regarded as the structural backbone of the brain network and involved in sleep. ${ }^{54,55}$ Sleep deprivation increased the reaction times and lapses rate, which has been attributed to less-efficient communication between brain areas ${ }^{56,57}$ and to changes in activity in the cingulate cortex, ${ }^{58,59}$ and which impaired the directed information flow of cingulate connectivity, proportional to its deleterious effect on vigilance. ${ }^{60} \mathrm{In}$ support of these findings, in our study we found that compared with GSs, PCPIs showed lower ReHo in the bilateral cingulate cortex with significant negative correlation with HAMA. Our findings suggest that disturbed nocturnal sleep may have a harmful effect on the cingulate cortex, which is involved in sleep and negative depression mood, and may be a key reason for post-insomnia negative mood.

\section{Lack of hippocampal cortex deficits}

Previous studies did not find any significant GMC, GMV, and white matter volume differences between PCPIs and GSs. ${ }^{15,18,19}$ Noh et $\mathrm{al}^{46}$ found that PCPIs displayed less absolute hippocampal volume (HV) and intracranial volume than GSs, but the differences between groups were not significant. They found that, in PCPIs, right and left HVs were negatively correlated with the duration of insomnia and the arousal index, and positively correlated with the recognition of visual memory. In addition, free recall in verbal memory was positively correlated with left $\mathrm{HV}$ in PCPIs. They thought that a long duration of insomnia and poor sleep quality contributed to a bilateral reduction in $\mathrm{HV}$. However, Riemann et $\mathrm{al}^{17}$ found that PCPIs demonstrated significantly reduced HV bilaterally compared with GSs. Notably, still left unclear is whether HV changed in PCPIs. In support of these findings, in the present study we further confirmed whether there are underlying ReHo brain-activity deficits in the parahippocampal gyrus. In our study, we did not find reduced ReHo areas in the hippocampus in all PCPIs compared with all GSs, and in PCPI-Ms compared with GS-Ms. However, few HVs were found in the higher $\mathrm{ReHo}$ in the right cluster of the posterior cingulate, occipital lobe, and parahippocampal gyrus in PCPI-Fs compared with GS-Fs.

Previous physiological, neuroimaging, and neurocognitive models demonstrated ruminative, hypervigilant, and/or excessive hyperarousal in CPI, which may relate to an imbalance of excitatory and inhibitory CNS influences. ${ }^{13,42,44}$ These studies provide supported evidence that the hyperarousal model was regarded as a core predisposing or perpetuating factor of CPI. ${ }^{61}$ This excess arousal is proposed to be manifested as exaggerated cortical, somatic, and cognitive activation, which leads to increased sensory and information processing, ultimately hampering the ability to initiate or maintain sleep. ${ }^{62,63}$ In support of these findings, the higher ReHo in small volumes of the posterior cingulate, occipital lobe, and parahippocampal gyrus was consistent with the excessive hyperarousal reactivity of CPI.

\section{Cerebellum deficits}

This is a crucial implication in light of mounting evidence for cerebellar involvement in various neurologic and psychiatric conditions, including autism, ${ }^{64}$ depression, ${ }^{65}$ and mood disorders. ${ }^{66}$ The cerebellum, to form a feedforward loop through the thalamus and to form a feedback loop through the pons, interconnects a network with extensive cortical and subcortical areas. These anatomic connections of extensive cortical and subcortical areas support the role of the cerebellum in cognitive and emotional processing. A large body of empirical research in patients with cerebellar damage has demonstrated that the region is related to emotional regulation. ${ }^{67,68}$ The cerebellum anterior lobe, which receives the nociceptive afferent fibers of spinal cord, is unconspicuous in cognitive and behavioral impairment once it is damaged. ${ }^{69}$ In our study, lower ReHo in the cerebellum anterior lobe was found both in PCPIs compared with GSs and in PCPI-Fs compared with GS-Fs. This finding suggests that disturbed nocturnal sleep may have a harmful effect on the cerebellar area and may imply the potential relationship between cerebellum and disturbed negative mood state in PCPIs.

\section{Conclusion}

In summary, our study demonstrated that the ReHo analysis, a useful noninvasive imaging tool for the detection of cerebral ReHo changes in PCPIs, could be useful for indexing the extent of insomnia, duration of insomnia, and mood state, and may be an early biomarker for detecting the altered brain activity in PCPIs. Multiple brain-region dysfunctions were involved in PCPIs, and significantly disturbed subjective sleep estimates as measured by PSQI and SRSS, and 
disturbed negative mood state as measured by the HAMD, HAMA, and POMS were found. The abnormal spontaneous neuronal activity in those areas provides information on the neural mechanisms underlying emotional and sleep-quality impairment in PCPIs.

\section{Acknowledgments}

The authors are grateful to the subjects who participated in this research. This work was supported by Jiangxi Provincial Department of Science and Technology Support Program (grant No 20132BBG70061 and No 20141BBG70026), Jiangxi Provincial Department of Natural Science Foundation Project (grant No 20132BAB205100), Jiangxi Provincial Department of Graduate Innovation Foundation (grant No YC2013-S007), Chinese Department of National Innovation Experiment Program for University Students (grant No 201210403052 and No 2012181).

\section{Disclosure}

This was not an industry-supported study. The authors have indicated no financial conflicts of interest in this work. None of the authors have any personal relationships, financial support, or involvement with organizations that hold financial interest in the subject matter. The authors declare no other conflicts of interest in this work.

\section{References}

1. Reimund E. The free radical flux theory of sleep. Med Hypotheses. 1994;43(4):231-233.

2. Luyster FS, Strollo PJ, Zee PC, Walsh JK; Boards of Directors of the American Academy of Sleep Medicine and the Sleep Research Society. Sleep: a health imperative. Sleep. 2012;35(6):727-734.

3. Whitton S. Nighty night sleep tight - health benefits of a good night's sleep. Insight. 2012;37(4):22.

4. Edinger JD, Means MK, Carney CE, Krystal AD. Psychomotor performance deficits and their relation to prior nights' sleep among individuals with primary insomnia. Sleep. 2008;31(5):599-607.

5. Altena E, Van Der Werf YD, Strijers RL, Van Someren EJ. Sleep loss affects vigilance: effects of chronic insomnia and sleep therapy. J Sleep Res. 2008;17(3):335-343.

6. Varkevisser M, Kerkhof GA. Chronic insomnia and performance in a 24-h constant routine study. J Sleep Res. 2005;14(1):49-59.

7. Carotenuto M, Esposito M, Parisi L, et al. Depressive symptoms and childhood sleep apnea syndrome. Neuropsychiatr Dis Treat. 2012;8: 369-373.

8. Johnson EO, Breslau N. Sleep problems and substance use in adolescence. Drug Alcohol Depend. 2001;64(1):1-7.

9. Liu X, Zhou H. Sleep duration, insomnia and behavioral problems among Chinese adolescents. Psychiatry Res. 2002;111(1):75-85.

10. Esposito M, Antinolfi L, Gallai B, et al. Executive dysfunction in children affected by obstructive sleep apnea syndrome: an observational study. Neuropsychiatr Dis Treat. 2013;9:1087-1094.

11. Morin CM, Benca R. Chronic insomnia. Lancet. 2012;379(9821): 1129-1141.

12. Roth T, Roehrs T, Pies R. Insomnia: pathophysiology and implications for treatment. Sleep Med Rev. 2007;11(1):71-79.
13. Nofzinger EA, Buysse DJ, Germain A, Price JC, Miewald JM, Kupfer DJ. Functional neuroimaging evidence for hyperarousal in insomnia. $\mathrm{Am}$ J Psychiatry. 2004;161(11):2126-2128.

14. Smith MT, Perlis ML, Chengazi VU, et al. Neuroimaging of NREM sleep in primary insomnia: a Tc-99-HMPAO single photon emission computed tomography study. Sleep. 2002;25(3):325-335.

15. Joo EY, Noh HJ, Kim JS, et al. Brain gray matter deficits in patients with chronic primary insomnia. Sleep. 2013;36(7):999-1007.

16. Neylan TC, Mueller SG, Wang Z, et al. Insomnia severity is associated with a decreased volume of the CA3/dentate gyrus hippocampal subfield. Biol Psychiatry. 2010;68(5):494-496.

17. Riemann D, Voderholzer U, Spiegelhalder K, et al. Chronic insomnia and MRI-measured hippocampal volumes: a pilot study. Sleep. 2007; 30(8):955-958.

18. Spiegelhalder K, Regen W, Baglioni C, et al. Insomnia does not appear to be associated with substantial structural brain changes. Sleep. 2013; 36(5):731-737.

19. Winkelman JW, Benson KL, Buxton OM, et al. Lack of hippocampal volume differences in primary insomnia and good sleeper controls: an MRI volumetric study at 3 Tesla. Sleep Med. 2010;11(6):576-582.

20. Buzsáki G, Draguhn A. Neuronal oscillations in cortical networks. Science. 2004;304(5679):1926-1929.

21. Zhang Z, Liu Y, Jiang T, et al. Altered spontaneous activity in Alzheimer's disease and mild cognitive impairment revealed by Regional Homogeneity. Neuroimage. 2012;59(2):1429-1440.

22. Tononi G, McIntosh AR, Russell DP, Edelman GM. Functional clustering: identifying strongly interactive brain regions in neuroimaging data. Neuroimage. 1998;7(2):133-149.

23. Zang Y, Jiang T, Lu Y, He Y, Tian L. Regional homogeneity approach to fMRI data analysis. Neuroimage. 2004;22(1):394-400.

24. Dai XJ, Gong HH, Wang YX, et al. Gender differences in brain regional homogeneity of healthy subjects after normal sleep and after sleep deprivation: a resting-state fMRI study. Sleep Med. 2012;13(6):720-727.

25. Peng DC, Dai XJ, Gong HH, et al. Altered intrinsic regional brain activity in male patients with severe obstructive sleep apnoea: a resting-state fMRI study. Neuropsychiatr Dis Treat. 2014;10:1819-1826.

26. Luo Y, Huang X, Yang Z, Li B, Liu J, Wei D. Regional homogeneity of intrinsic brain activity in happy and unhappy individuals. PLoS One. 2014;9(1):e85181.

27. Qiu C, Liao W, Ding J, et al. Regional homogeneity changes in social anxiety disorder: a resting-state fMRI study. Psychiatry Res. 2011; 194(1): $47-53$.

28. Lai $\mathrm{CH}, \mathrm{Wu} \mathrm{YT}$. Changes in regional homogeneity of parieto-temporal regions in panic disorder patients who achieved remission with antidepressant treatment. J Affect Disord. 2013;151(2):709-714.

29. Philip NS, Kuras YI, Valentine TR, et al. Regional homogeneity and resting state functional connectivity: associations with exposure to early life stress. Psychiatry Res. 2013;214(3):247-253.

30. Liang MJ, Zhou Q, Yang KR, et al. Identify changes of brain regional homogeneity in bipolar disorder and unipolar depression using restingstate FMRI. PLoS One. 2013;8(12):e79999.

31. Buysse DJ, Reynolds CF 3rd, Monk TH, Berman SR, Kupfer DJ. The Pittsburgh Sleep Quality Index: a new instrument for psychiatric practice and research. Psychiatry Res. 1989;28(2):193-213.

32. Bastien CH, Vallières A, Morin CM. Validation of the Insomnia Severity Index as an outcome measure for insomnia research. Sleep Med. 2001; 2(4):297-307.

33. Hamilton M. A rating scale for depression. J Neurol Neurosurg Psychiatry. 1960;23(1):56-62.

34. Hamilton M. The assessment of anxiety states by rating. $\mathrm{Br} J \mathrm{Med}$ Psychol. 1959;32(1):50-55.

35. McNair DM, Lorr M, Droppleman LM. Manual for the Profile of Mood States. San Diego, CA: Educational and Industrial Testing Services; 1971.

36. McNair DM, Lorr M, Droppleman LF. EdITS manual for the Profile of Mood States. San Diego, CA: EdITS Educational and Industrial Testing Service; 1992. 
37. Satterthwaite TD, Elliott MA, Gerraty RT, et al. An improved framework for confound regression and filtering for control of motion artifact in the preprocessing of resting-state functional connectivity data. Neuroimage. 2013;64:240-256.

38. Yan CG, Cheung B, Kelly C, et al. A comprehensive assessment of regional variation in the impact of head micromovements on functional connectomics. Neuroimage. 2013;76:183-201.

39. Fox MD, Snyder AZ, Vincent JL, Corbetta M, Van Essen DC, Raichle ME. The human brain is intrinsically organized into dynamic, anticorrelated functional networks. Proc Natl Acad Sci. 2005; 102(27):9673-9678.

40. Guo W, Jiang J, Xiao C, et al. Decreased resting-state interhemispheric functional connectivity in unaffected siblings of schizophrenia patients. Schizophr Res. 2014;152(1):170-175.

41. Saad ZS, Gotts SJ, Murphy K, et al. Trouble at rest: how correlation patterns and group differences become distorted after global signal regression. Brain Connect. 2012;2(1):25-32.

42. Harvey AG. A cognitive model of insomnia. Behav Res Ther. 2002; 40(8):869-893.

43. Morin CM. Insomnia: Psychological Assessment and Management. New York, NY: The Guilford Press; 1993.

44. Merica H, Blois R, Gaillard JM. Spectral characteristics of sleep EEG in chronic insomnia. Eur J Neurosci. 1998;10(5):1826-1834.

45. Altena E, Vrenken H, Van Der Werf YD, van den Heuvel OA, Van Someren EJ. Reduced orbitofrontal and parietal gray matter in chronic insomnia: a voxel-based morphometric study. Biol Psychiatry. 2010; 67(2):182-185.

46. Noh HJ, Joo EY, Kim ST, et al. The relationship between hippocampal volume and cognition in patients with chronic primary insomnia. J Clin Neurol. 2012;8(2):130-138.

47. Davidson RJ, Abercrombie H, Nitschke JB, Putnam K. Regional brain function, emotion and disorders of emotion. Curr Opin Neurobiol. 1999; 9(2):228-234.

48. Phillips ML, Drevets WC, Rauch SL, Lane R. Neurobiology of emotion perception I: The neural basis of normal emotion perception. Biol Psychiatry. 2003;54(5):504-514.

49. Cardoso EF, Maia FM, Fregni F, et al. Depression in Parkinson's disease: convergence from voxel-based morphometry and functional magnetic resonance imaging in the limbic thalamus. Neuroimage. 2009; 47(2):467-472.

50. Ring HA, Bench CJ, Trimble MR, Brooks DJ, Frackowiak RS, Dolan RJ. Depression in Parkinson's disease. A positron emission study. Br J Psychiatry. 1994;165(3):333-339.

51. Terroni L, Amaro E, Iosifescu DV, et al. Stroke lesion in cortical neural circuits and post-stroke incidence of major depressive episode: a 4-month prospective study. World J Biol Psychiatry. 2011;12(7):539-548.

52. Szily E, Kéri S. Emotion-related brain regions. Ideggyogy Sz. 2008; 61(3-4):77-86.

53. Allman JM, Hakeem A, Erwin JM, Nimchinsky E, Hof P. The anterior cingulate cortex. The evolution of an interface between emotion and cognition. Ann N Y Acad Sci. 2001;935:107-117.
54. Piantoni G, Poil SS, Linkenkaer-Hansen $\mathrm{K}$, et al. Individual differences in white matter diffusion affect sleep oscillations. J Neurosci. 2013; 33(1):227-233.

55. Murphy M, Riedner BA, Huber R, Massimini M, Ferrarelli F, Tononi G. Source modeling sleep slow waves. Proc Natl Acad Sci U S A. 2009; 106(5):1608-1613.

56. Chee MW, Tan JC, Zheng H, et al. Lapsing during sleep deprivation is associated with distributed changes in brain activation. J Neurosci. 2008;28(21):5519-5528.

57. Sadaghiani S, Scheeringa R, Lehongre K, Morillon B, Giraud AL, Kleinschmidt A. Intrinsic connectivity networks, alpha oscillations, and tonic alertness: a simultaneous electroencephalography/ functional magnetic resonance imaging study. J Neurosci. 2010; 30(30):10243-10250.

58. Gujar N, Yoo SS, Hu P, Walker MP. The unrested resting brain: sleep deprivation alters activity within the default-mode network. $J \operatorname{Cog} n$ Neurosci. 2010;22(8):1637-1648.

59. Tomasi D, Wang RL, Telang F, et al. Impairment of attentional networks after 1 night of sleep deprivation. Cereb Cortex. 2009;19(1): 233-240.

60. Piantoni G, Cheung BL, Van Veen BD, et al. Disrupted directed connectivity along the cingulate cortex determines vigilance after sleep deprivation. Neuroimage. 2013;79:213-222.

61. Perlis ML, Smith MT, Pigeon WR. Etiology and pathophysiology of insomnia. In: Kryger MH, Roth T, Dement WC, editors. Principles and Practice of Sleep Medicine. 4th ed. Philadelphia, PA: Elsevier Inc.; 2005:714-725

62. Riemann D, Spiegelhalder K, Feige B, et al. The hyperarousal model of insomnia: a review of the concept and its evidence. Sleep Med Rev. 2010;14(1):19-31.

63. Perlis ML, Merica H, Smith MT, Giles DE. Beta EEG activity and insomnia. Sleep Med Rev. 2001;5(5):363-374.

64. Allen G, Courchesne E. Differential effects of developmental cerebellar abnormality on cognitive and motor functions in the cerebellum: an fMRI study of autism. Am J Psychiatry. 2003;160(2):262-273.

65. Liu Z, Xu C, Xu Y, et al. Decreased regional homogeneity in insula and cerebellum: a resting-state fMRI study in patients with major depression and subjects at high risk for major depression. Psychiatry Res. 2010;182(3):211-215.

66. Soares JC, Mann JJ. The anatomy of mood disorders - review of structural neuroimaging studies. Biol Psychiatry. 1997;41(1):86-106.

67. Parvizi J, Anderson SW, Martin CO, DamasioH,Damasio AR. Pathological laughter and crying: a link to the cerebellum. Brain. 2001;124(Pt 9): 1708-1719.

68. Schmahmann JD, Sherman JC. The cerebellar cognitive affective syndrome. Brain. 1998;121(Pt 4):561-579.

69. Paulus KS, Magnano I, Conti M, et al. Pure post-stroke cerebellar cognitive affective syndrome: a case report. Neurol Sci. 2004;25(4): $220-224$.
Neuropsychiatric Disease and Treatment

\section{Publish your work in this journal}

Neuropsychiatric Disease and Treatment is an international, peerreviewed journal of clinical therapeutics and pharmacology focusing on concise rapid reporting of clinical or pre-clinical studies on a range of neuropsychiatric and neurological disorders. This journa is indexed on PubMed Central, the 'PsycINFO' database and CAS,

\section{Dovepress}

and is the official journal of The International Neuropsychiatric Association (INA). The manuscript management system is completely online and includes a very quick and fair peer-review system, which is all easy to use. Visit http://www.dovepress.com/testimonials.php to read real quotes from published authors. 